\title{
Addition of silica nanoparticles to tailor the mechanical properties of nanofibrillated cellulose thin films
}

Mohamed Eita, Hans Arwin, Hjalmar Granberg and Lars Wagberg

\section{Linköping University Post Print}

N.B.: When citing this work, cite the original article.

Original Publication:

Mohamed Eita, Hans Arwin, Hjalmar Granberg and Lars Wagberg, Addition of silica nanoparticles to tailor the mechanical properties of nanofibrillated cellulose thin films, 2011, Journal of Colloid and Interface Science, (363), 2, 566-572.

http://dx.doi.org/10.1016/j.jcis.2011.07.085

Copyright: Elsevier http://www.elsevier.com/

Postprint available at: Linköping University Electronic Press

http://urn.kb.se/resolve?urn=urn:nbn:se:liu:diva-71372 


\title{
Addition of silica nanoparticles to tailor the
}

\section{mechanical properties of nanofibrillated cellulose}

\section{thin films}

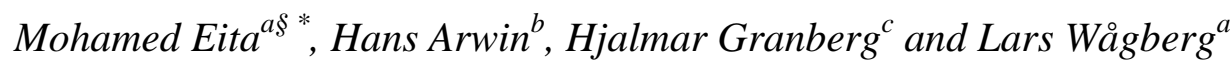

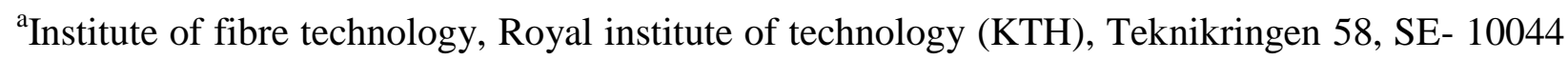
Stockholm, Sweden. ${ }^{b}$ Laboratory of Applied Optics, Linköping University, SE-58183, Sweden. ${ }^{\mathrm{c}}$ Innventia AB, Box 5604, SE-114 86 Stockholm

* Author to whom correspondence may be addressed. ${ }^{\S}$ Current Address: Division of functional materials (FNM), Royal institute of technology (KTH), 16440 Kista, Sweden, Tel. 00468 7908148, e-mail: eita@kth.se.

Keywords: Buckling, Cellulose, Light transmission, Porous, Strain, Thin film.

\begin{abstract}
Over the last decade, the use of nanocellulose in advanced technological applications has been promoted both due the excellent properties of this material in combination with its renewability. In this study, multilayered thin films composed of nanofibrillated cellulose (NFC), polyvinyl amine (PVAm) and silica nanoparticles were fabricated on polydimethylsiloxane (PDMS) using a layer-by-layer adsorption technique. The multilayer build-up was followed in situ by quartz crystal microbalance with dissipation, which indicated that the $\mathrm{PVAm}_{-} \mathrm{SiO}_{2}-\mathrm{PVAm}-\mathrm{NFC}$ system adsorbs twice as much wet mass material compared to the PVAm-NFC system for the same number of bilayers. This is accompanied with a higher viscoelasticity for the $\mathrm{PVAm}-\mathrm{SiO}_{2}$ PVAm-NFC system. Ellipsometry indicated a dry-state thickness of 2.2 and $3.4 \mathrm{~nm}$ per bilayer
\end{abstract}


for the PVAm-NFC system and the PVAm-SiO $2-\mathrm{PVAm}-\mathrm{NFC}$ system, respectively. Atomic force microscopy height images indicate that in both systems, a porous network structure is achieved. Young's modulus of these thin films was determined by the Strain-Induced Elastic Buckling Instability for Mechanical Measurements (SIEBIMM) technique. The Young's modulus of the PVAm/NFC films was doubled, from 1 to $2 \mathrm{GPa}$, upon incorporation of silica nanoparticles in the films. The introduction of the silica nanoparticles lowered the refractive index of the films, most probably due to an increased porosity of the films.

\section{Introduction}

Today there is a large interest in using Cellulose NanoCrystals (CNC) and NanoFibrillated Cellulose (NFC) as building blocks in new materials [1,2]. There are naturally several reasons to this but the development of the nanotechnology where nano-sized building blocks can be used to construct small, interactive devices in combination with the need for renewable materials has naturally contributed to this interest. It has for example been shown that the CNC can selforganize [2] to give a chiral nematic arrangement of the CNC film as water is evaporated from the CNC dispersion. It has also been shown that both CNC and NFC can be incorporated into Layer-by-Layer (LbL) self assemblies to yield films with controlled thickness and hence controlled optical properties depending on the composition of the film [3,4]. Recent investigations have also shown that it is possible to prepare freestanding films on NFC $[5,6]$ with controlled barrier properties and with very low oxygen permeabilities at low relative humidities (RH). Another very interesting property of the NFC material is the very good mechanical properties of films and nano-papers prepared from this material [7] and free-standing LbL's from NFC and Poly EthyleneImine (PEI) [8].

All these different examples show that CNC and NFC are very interesting materials for the preparation of new nano-composites with excellent mechanical properties and controlled optical 
and permeability properties. It is, though, very difficult to evaluate the properties of nanometer sized films due to handling properties of these types of free-standing films [8]. However, following the development of the use of Strain Induced Elastic Buckling Instability for Mechanical Measurements (SIEBIMM) [9-12] it was recently shown that it is possible to use the SIEBIMM technology to evaluate the mechanical properties of nanometer sized films of NFC and PEI [13]. Furthermore, by using high resolution optical methods [14], it should also be possible to determine the optical properties of thin films deposited on the same types of supports (i.e PolyDiMethylSiloxane) (PDMS) as used for the SIEBIMM technology.

Determination of the Young's modulus of thin films has been achieved by a Strain-induced elastic buckling instability for mechanical measurements (SIEBIMM) technique [9]. This technique is based on the buckling occurring in a thin film of a higher Young's modulus attached to an elastic lower modulus substrate when subjected to a in plane compression. To relieve the strain the thin film will buckle [10]. The buckling of thin film can be used to determine Young's modulus $E_{f}$ of that film according to [11]:

$$
E_{f}=\mathbf{3} E_{s} \frac{\left(1-v_{f}^{2}\right)}{\left(1-v_{s}^{2}\right)}\left[\frac{\lambda}{2 \pi d_{f}}\right]^{3}
$$

where $E_{s}$ is Young's modulus of the substrate, $v_{f}$ and $v_{s}$ are Poisson's ratios of the film and the substrate, usually assumed to be 0.33 and 0.5 , respectively [9-10], $\lambda$ is the buckling wavelength determined from the buckling measurements, and $d_{f}$ is the film thickness. Thus, the most critical parameters in the SIEBIMM technique that determines Young's modulus of a thin film are the buckling wavelength $\lambda$ and the film thickness $d_{f}$. Buckling of a thin film on an elastic Polydimethylsiloxane (PDMS) substrate can be achieved by a mechanical compression or thermal induction [12]. Whereas the thickness of polymer thin films increases with increasing relative humidity, due to moisture uptake, the Young's modulus of the film was found to 
decrease with increasing relative humidity [15]. SIEBIMM has been applied to different systems varying from polymer self-assembled multilayers and composites [16-19], semiconductors [20], metals [21] and carbon nanotubes [22].

Quartz crystal microbalance with dissipation (QCM-D) is an acoustic wave based technique. It monitors both frequency and dissipation shifts due to surface processes like adsorption or contact with liquids. Dissipation $D$ is expressed in terms of the oscillating frequency $f$ of the crystal and its decay time $\tau$ as $D=1 / \pi \tau f$ [23]. The mass of the adsorbed film in QCM-D measurements can be determined by the Sauerbrey equation [24].

$$
\Delta f_{N}=-N \frac{2 f_{0}^{2}}{v_{s} \rho_{q}} \Delta m
$$

where $\Delta f_{N}$ is the change of resonance frequency at the $N$ th overtone, $N=1,3,5 \ldots$, and $v_{s}$ (the shear wave velocity $)=3340 \mathrm{~m} \mathrm{~s}^{-1}, f_{0}$ (the fundamental crystal frequency $)=5 \mathrm{MHz}$ for an AT-cut quartz, $\rho_{q}$ is the density of quartz $=2.65 \mathrm{~g} \mathrm{~cm}^{-3}$, and $\Delta m$ is the adsorbed mass per unit area. By substituting these values in equation $3, \Delta m$ can be written as: $\Delta m=-C \Delta f_{N} / N$, where $C=0.177$ $\mathrm{mg} \mathrm{m}^{-2} \mathrm{~Hz}^{-1}$. However, this requires a no-slip condition, which means that the adlayer is rigidly attached and shows almost no contribution of the viscoelastic effects to the frequency shift, and hence the dissipation shift $(\Delta D)$ should be close to zero [25-26]. In addition to determining the adsorbed masses, QCM-D is also efficient in studying the viscoelastic properties of the adsorbed layer [27]. Combined with other techniques such as ellipsometry or X-ray photoelectron spectroscopy, QCM-D was applied to determine the adlayer structure with respect to the binding sites, the final layer thickness or the internal layer structure [28].

In the present work it has been the aim to evaluate how the composition of LbLs of NFCs and different polyelectrolytes and nano-colloids will influence the mechanical and optical properties of nanometer thin films of NFC. It has hence been our objective to develop functional materials based on renewable NFC by building up thin films of NFC, PolyVinylAmine (PVAm), and $\mathrm{SiO}_{2}$ 
nanoparticles. The substrate used was PDMS, which is known for its elasticity and thus is essential for the SIEBIMM method. It is also known for its high transmittance of visible light which allows for a reliable evaluation of the optical properties of the films formed on top of the PDMS. By using nano-silica in combination with NFC and different types of polyelectrolytes it was also the aim to form films with controlled porosities and controlled mechanical and optical properties. In order to follow the buildup and internal structure of the different material combinations we used Quartz Crystal Microbalance with Dissipation (QCM-D), Atomic force microscopy (AFM) and variable angle spectroscopic ellipsometry to evaluate the properties of the formed films.

\section{Experimental}

Materials: PVAm, Lupamine 5095 BASF, 5-10\% aqueous solution, M.W. 45000, Poly(allylamine hydrochloride), (PAH), M.W. 70000, was purchased from Aldrich, Germany. NFC was prepared at Innventia AB, Sweden. Silica nanoparticles dispersion of average size of $5 \mathrm{~nm}$ was purchased from EKA, Sweden. PDMS, as a base and curing reagent, brand SYLGARD 184, was purchased from Chem Tech AB, Sweden.

Preparation of multilayers: Solutions of PAH and NFC were prepared at concentration of 100 $\mathrm{mg} / \mathrm{L}$ in ultrapure water, whereas PVAm, was prepared at a concentration of $20 \mathrm{mg} / \mathrm{L}$, Silica nanoparticles was diluted to $0.01 \%$ in ultrapure water and $\mathrm{pH}$ adjusted with $0.1 \mathrm{M} \mathrm{HCl}$ from 9 to 6,6. NFC and PVAm had a pH of 6.6 upon dilution. PDMS substrates were prepared by mixing the base to the curing reagent at a ratio of 10:1. The mixture was stirred very well in order to achieve homogeneity and was then poured onto a casting mould made of glass and having a basement of silica. To eliminate bubbles suspended into the casted PDMS, it was placed in a dessicator under vacuum for a few minutes and finally heated in oven to $100^{\circ} \mathrm{C}$ for 2 hours to cross link. PDMS was cut into $5 \times 1 \mathrm{~cm}$ stripes and sonicated in $2 \%$ sodium dodecyl sulphate SDS solution for $15 \mathrm{~min}$ and in ultrapure water for $30 \mathrm{~min}$ prior to use. Multilayers were prepared 
using the layer-by-layer technique with a robot from Nanostrata Inc. USA. PDMS substrates were dipped in solutions according to the following timing scheme: $20 \mathrm{~min}$ for the precursor layer of PAH, 15 min for NFC, 10 min for PVAm. In case of PVAm-NFC films, 3 rinsing steps in ultrapure water for 2 min each were applied after each layer, in case of PVAm-SiO $2-\mathrm{PVAm}$ NFC films, only one rinsing step for 3 min after PVAm or 5 min after NFC or $\mathrm{SiO}_{2}$ were applied.

Characterization: QCM-D measurements were performed using the E4 equipment supplied by Q-sense, Sweden. AT-Cut silicon dioxide-coated quartz crystals of $5 \mathrm{MHz}$ initial frequency were used. Circulation of liquids through the measuring chamber was done by a peristaltic pump at a constant rate of $150 \mu \mathrm{L} / \mathrm{min}$. To coat a PDMS layer on the $\mathrm{SiO}_{2}$ crystal, PDMS was prepared by adding the base to the curing agent in a ration of 10:1. This mixture was then dissolved in Hexane at a concentration of $0.5 \%$ (wt.) and stirred until a clear solution was obtained. $\mathrm{A} \mathrm{SiO}_{2}$ crystal was rinsed by water and ethanol and then plasma treated for 3 minutes. The crystal sensing surface was spin coated with a PDMS thin film at a speed of $2000 \mathrm{rpm}$. The thickness of a PDMS thin film on $\mathrm{SiO} 2$ wafer is about $45 \mathrm{~nm}$ as measured by ellipsometry. The PDMScoated $\mathrm{SiO}_{2}$ crystal was placed in the oven at $100^{\circ} \mathrm{C}$ for 2 hours for crosslinking.

Thicknesses of NFC multilayers on PDMS stripes were measured with a variable angle spectroscopic ellipsometer of the type J. A. Wollam Co. USA, using the angle range of 50-70 $\mathrm{C}$. Ellipsometric data were fitted to a Cauchy model to get the thickness of multilayers. AFM imaging was done using a Nanoscope Multimode Шa microscope, from digital instruments, Santa Barbara, CA, USA. Images were taken in tapping mode at ambient conditions, of $23^{\circ} \mathrm{C}$ and $50 \%$ humidity. N-doped silica cantilevers of a spring constant of 20-80 N/m were used.

Transmission measurements were performed using a Perkin Elmer lambda-750 spectrometer in the wavelength range of 200-800 nm. To determine Young's modulus of multilayers, a micro tester compartment from DEBEN Ltd. UK, was used which has a load cell of 200 N. A PDMS 
stripe having multilayers on top was inserted in the micro tester and fixed by screws. Compression force are applied gradually on the PDMS to produce buckling which is monitored in situ by an optical microscope at a magnification of 50 times. The applied force ranged from 0 to $1.5 \mathrm{~N}$. Sample strain was measured by a calibre and did not exceed $0.5 \mathrm{~mm}$ which is $1 \%$ of the sample length. Mechanical testing measurements were performed at ambient conditions; about $53 \%$ humidity and $23^{\circ} \mathrm{C}$. Images were captured and analyzed using the software "vision assistant" supplied by National Instruments.

\section{Results and discussion}

Thin films were prepared on PDMS-coated $\mathrm{SiO}_{2}$ substrates using a layer-by-layer sequence and monitored in situ by QCM-D (figure 1). First, a PAH precursor layer was adsorbed directly on the PDMS substrate to provide a positive charge to the PDMS substrate and to reduce its hydrophobicity. An NFC layer was also adsorbed before multilayers were built in sequence PVAm-NFC (figure 1a) or PVAm-SiO 2 -PVAm-NFC (figure 1b). Noticeably, the precursor PAH layer had a very limited frequency shift in both systems indicating very low adsorption, which can be attributed to the hydrophobicity and lack of charge of the PDMS substrate. However, it provides enough positive charge for the next NFC layer to adsorb and hence allows the upcoming oppositely charged layers to adsorb regularly. Frequency and dissipation shifts increase with increasing the number of layers in both systems as a result of the charge and coverage provided by these layers. PVAm usually causes lower frequency and dissipation shifts, whereas those of NFC and $\mathrm{SiO} 2$ are much higher and close to each other. Single dissipation shifts for each $\mathrm{SiO}_{2}$ layer are less than those of NFC, which can be attributed to the size and shape of each of them. While NFC, as a fibril, could entangle and entrap more water, thus enhance viscoelasticity, $\mathrm{SiO}_{2}$ nanoparticles have a spherical shape and thus assemble on the fibril surface without allowing as much entrapped water or viscoelastic character. $\Delta D$ versus $\Delta f$ plots of both systems show linear increase of dissipation with increasing frequency (figure $2 a$ ). The 
PVAm-SiO 2 -PVAm-NFC system shows higher $\Delta D / \Delta f$ values than the PVAm-NFC system. This indicates that the PVAm-SiO 2 -PVAm-NFC system is more viscoelastic than the PVAm-NFC system, or in other words, the latter is more rigid. Apparently, the assembly of spheres and fibrils separated by the very thin PVAm layer may be the reason of such higher viscoelasticity values in the final layer structure. Adsorbed masses were calculated in $\mathrm{mg} / \mathrm{m}^{2}$ by the Sauerbrey equation (equation 2) and plotted against the number of single layers (figure 2b).
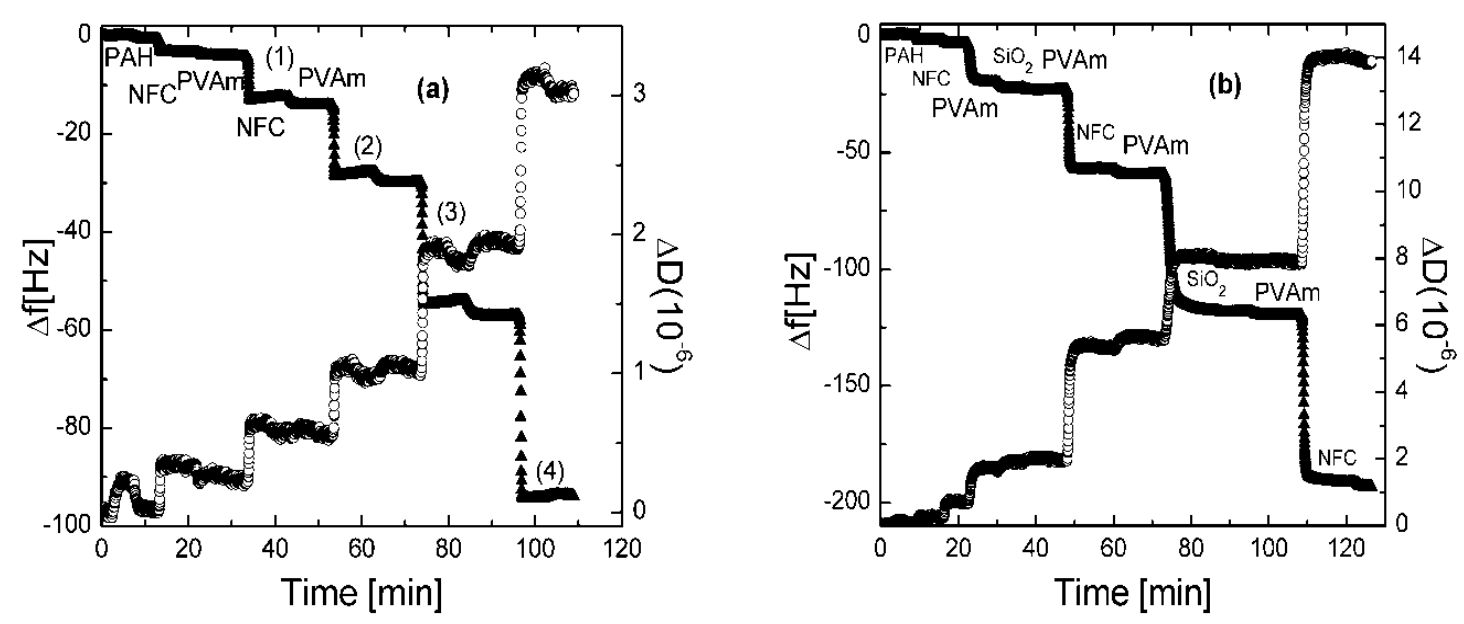

Fig. 1. Multilayer formation as monitored by QCM-D of (a) 4 bilayers of (PVAm-NFC) after an initial bilayer of (PAHNFC) and (b) 2 (PVAM-SiO ${ }_{2}$-PVAm-NFC) tetralayers after an initial bilayer of (PAH-NFC). $\Delta f$ and $\Delta D$ are presented as filled triangles and empty circles, respectively, data present the fifth overtone.

The same number of layers was built up on the substrate. However due to structural differences between the systems, the PVAm-NFC system has four bilayers and PVAm-SiO 2 -PVAm-NFC has two tetralayers, but both have a PAH-NFC initial bilayer. The PAH precursor layer has a very low mass due to the hydrophobicity and lack of charge as discussed above. In turn, the upcoming NFC layer also shows very limited mass due to the low coverage of the PAH layer. However, as the number of layers increases, the adsorbed mass of each component in both systems increases as well, so that the general adsorption pattern is described by increasing the adsorbed mass with increasing the number of layers. In a previous work [13], we have reported on the same behavior in the build-up of PEI/NFC multilayers on a PDMS. The adsorbed masses of NFC and PVAm in the NFC-PVAm system are 14.8 and $1.4 \mathrm{mg} / \mathrm{m}^{2}$ (calculated by Sauerbrey 
equation), respectively, corresponding to a total mass of $16.2 \mathrm{mg} / \mathrm{m}^{2}$, i.e. NFC represents $91 \%$ of the whole film. In case of PVAm-SiO $2-\mathrm{PVAm}-\mathrm{NFC}$ films; $\mathrm{NFC}, \mathrm{SiO}_{2}$ and $\mathrm{PVAm}$ have masses of $17.5,13.9$ and $1 \mathrm{mg} / \mathrm{m}^{2}$ out of a total mass of $32.4 \mathrm{mg} / \mathrm{m}^{2}$, i.e. both $\mathrm{NFC}$ and $\mathrm{SiO}_{2}$ represent about $97 \%$ of the whole film, whereas only NFC presents about $54 \%$ of the whole film. Imaging these films by AFM could integrate the picture made by QCM-D about the internal film structure.
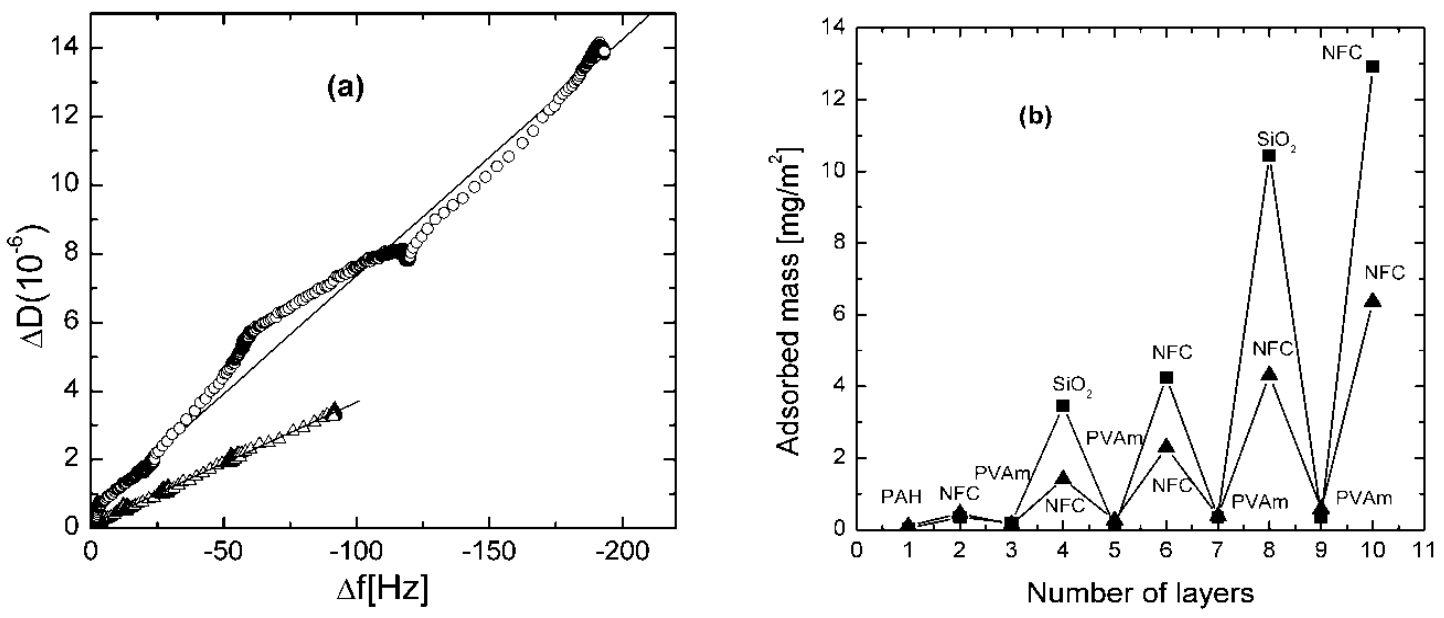

Fig. 2. (a) $\Delta D$ versus $\Delta f$ plot of the two systems (PVAM-SiO ${ }_{2}-\mathrm{PVAm}-\mathrm{NFC}$ ), circles, and (PVAm-NFC), triangles, taken from QCM-D measurements in figure 1.(b) the adsorbed masses of the two systems as calculated by Sauerbrey equation.

Figure 3 shows tapping mode AFM images of both systems as height, height profile and phase recorded on the PDMS-coated $\mathrm{SiO}_{2}$ crystal used in situ in the QCM-D measurements in figure 2. The RMS surface roughness measured is 1.9 and $3.6 \mathrm{~nm}$ for the PVAm-NFC and PVAm-SiO $2_{2}^{-}$ PVAm-NFC films, respectively. Considering that the adsorbed mass of the PVAm-SiO $2-\mathrm{PVAm}-$ NFC film is approximately twice as large compared to the PVAm-NFC film for the same amount of bilayers, it turns out that both films have approximately the same roughness at the same adsorbed mass. Surface roughness previously reported [13] for a film of 1.5 PAH (PEI-NFC) bilayers is $1.5 \mathrm{~nm}$, which is close to the current value of the PVAm-NFC system, considering the difference in number of layers. It is also clear that PVAm which is linear, yields less roughness than the branched PEI used in the previous study, assuming the same number of layers. PVAm 
introduces an interesting property in the film, which is nanoporosity. The AFM height images in figure 3 show that both systems contain deep holes corresponding to nanopores of different size distribution. This is attributed to the linearity of the PVAm chains as well as the size and shape difference of each component in the film. When the NFC fibrils adsorb on the surface, they allow spaces between the fibrils, they also entangle and may have different spatial conformations.
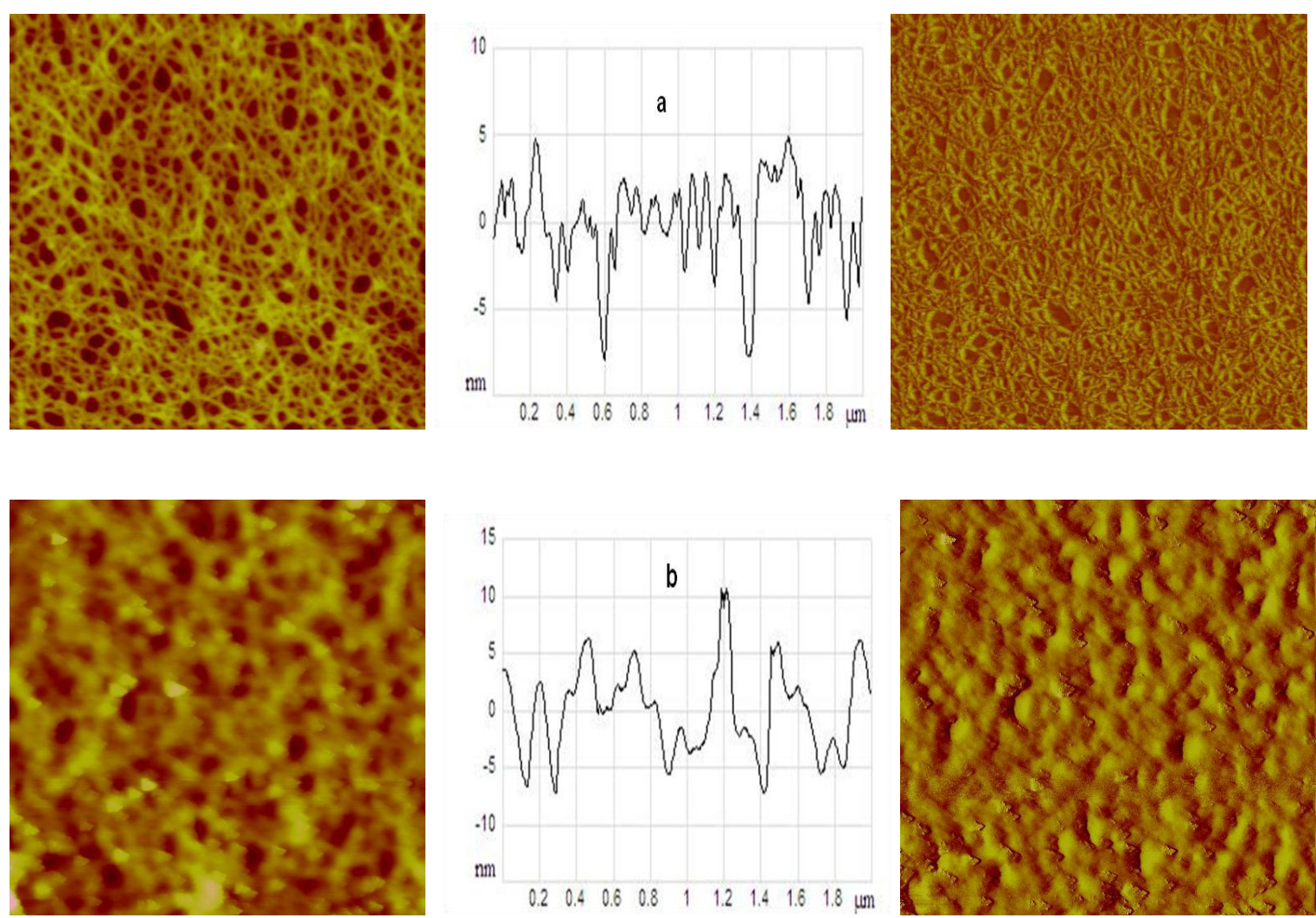

Figure 3. AFM height, height profile and phase images, from left to right respectively of (a) PVAm-NFC and (b) PVAm$\mathrm{SiO}_{2}$-PVAm-NFC on a PDMS-coated $\mathrm{SiO}_{2}$ crystals which were used in measurements in figure 1 .

When PVAm adsorbs on the surface, its positive charge compensates for the negative charge of NFC. However, the linearity of the PVAm chains does not allow higher conformational degree of freedom, which results in a smooth linear assembly of PVAm allowing voids between its chains. The subsequently adsorbing $\mathrm{SiO}_{2}$ nanoparticles or NFC, which are negatively charged, adsorb only where there are positive charges. This process continues so that the system maintains its porous structure with increasing number of layers. 
Thicknesses of the two types of films on PDMS substrates were measured by spectroscopic ellipsometry (figure 4). The number of layers is expressed in bilayers, where a PVAm-NFC cycle is one bilayer and each PVAm-SiO 2 -PVAm-NFC cycle consists of two bilayers. Over a range of 50 bilayers, film thickness develops linearly for both systems with a higher increment for PVAm-SiO 2 -PVAm-NFC than PVAm-NFC. The average thickness per bilayer is 2.2 and 3.4 nm for PVAm-NFC and PVAm-SiO 2 -PVAm-NFC films, respectively. The thickness difference is referred to the increased adsorption upon introducing $\mathrm{SiO}_{2}$ nanoparticles to the system. $\mathrm{SiO}_{2}$ nanoparticles enhance the adsorption of the upcoming layers by introducing hydrophilicity, charge and high coverage due to their spherical shape and small size. In the previous work on PEI/NFC multilayers on PDMS substrates [13], a thickness of $4 \mathrm{~nm}$ per bilayer was recorded by spectroscopic ellipsometry. This emphasizes the difference in adsorption between PEI and PVAm which is usually referred to as differences in the polyelectrolyte charge, the chain length or the molecular weight, the hydrophilic character and the chain structure, e.g. linear or branched chains. Among these differences, the chain structure may lead to a difference in the adsorbed amount as revealed from AFM images and ellipsometry thicknesses in the current work and the previous one [13]. Hence the branched PEI is supposed to afford more adsorption sites than the linear PVAm.

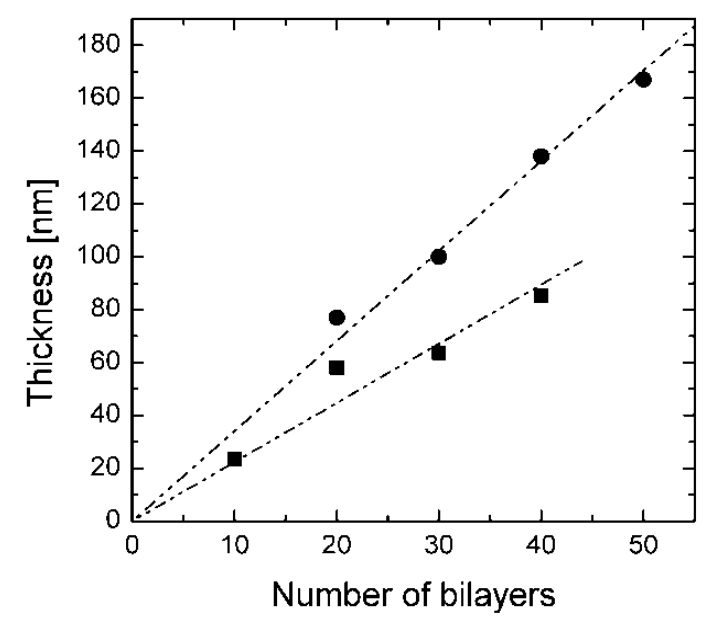

Fig. 4. Thicknesses of thin films of PVAm-NFC, squares, and PVAm-SiO - PVAm-NFC, circles, as measured by ellipsometry. Average thickness per bilayer is 2.2 and 3.4 nm for PVAm-NFC and PVAm-SiO 2 -PVAm-NFC, respectively. 
Young's modulus of PVAm-NFC and PVAm-SiO 2 -PVAm-NFC thin films was determined using the SIEBIMM technique. The Young's modulus of the PDMS substrate was determined as 1.9 MPa in the previous work [13]. The two critical parameters in determining the Young's modulus using SIEBIMM are the film thickness $d_{f}$ and the buckling wavelength $\lambda$. Similar to thickness development (figure 4), the buckling wavelengths show a linear propagation as well (figure 5a), with an increasing rate for PVAm-SiO 2 -PVAm-NFC than for PVAm-NFC. It has been reported elsewhere $^{1-5}$ that the buckling wavelength increases with increasing the number of layers. The Young' modulus, however, should not be affected by thickness over a certain thickness range [913] which is also demonstrated here in figure 5b. Young's modulus is calculated as 1.106 $( \pm 0.145) \mathrm{GPa}$ and $2.181( \pm$ 0.083) GPa for PVAm-NFC and PVAm-SiO $2-\mathrm{PVAm}-\mathrm{NFC}$ respectively. Consequently, the Young's modulus is doubled upon introducing $\mathrm{SiO}_{2}$ nanoparticles to the PVAm-NFC system. The Young's modulus of PEI-NFC thin films was determined previously as $1.5( \pm 0.2) \mathrm{GPa}$ [13]. The lower value reported here for the system PVAm-NFC may be attributed to the difference in the chain structure between PEI and PVAm (see discussion of figure 2). Whereas PEI is branched, PVAm is linear, thus the former could allow more adsorption of NFC in different directions which results in a less porous film. Other factors, such as the polyelectrolyte charge or hydrophilicity may affect the adsorption. A nanoindentation study showed that the elastic modulus of silica nanoparticles is $68.9 \mathrm{GPa}$ [29]. Due to their higher Young's modulus, silica nanoparticles have been used to enhance Young's modulus of polymeric materials [30]. Microporous silica thin films were also fabricated [31], these thin films exhibited a double effect of a low refractive index caused by porosity, and a higher Young's modulus of about 19.5 GPa. Thus silica nanoparticles have high potential to increase the strength of thin films as well as to introduce porosity. In the current system, PVAm$\mathrm{SiO}_{2}$-PVAm-NFC, introducing silica nanoparticles to the system does have an impact on Young's modulus of the whole film. However, the resulting Young's modulus is not within the same order of magnitude as that of the bulk silica nanoparticles. In fact, the Young's modulus of 
the whole film is naturally affected by the other components in the film, e.g. NFC, which yielded an order of magnitude lower Young's modulus of $6 \mathrm{GPa}$ [13]. The distribution of silica nanoparticles in the film as well as the porosity may result in a final value that is far lower than the value of bulk silica.
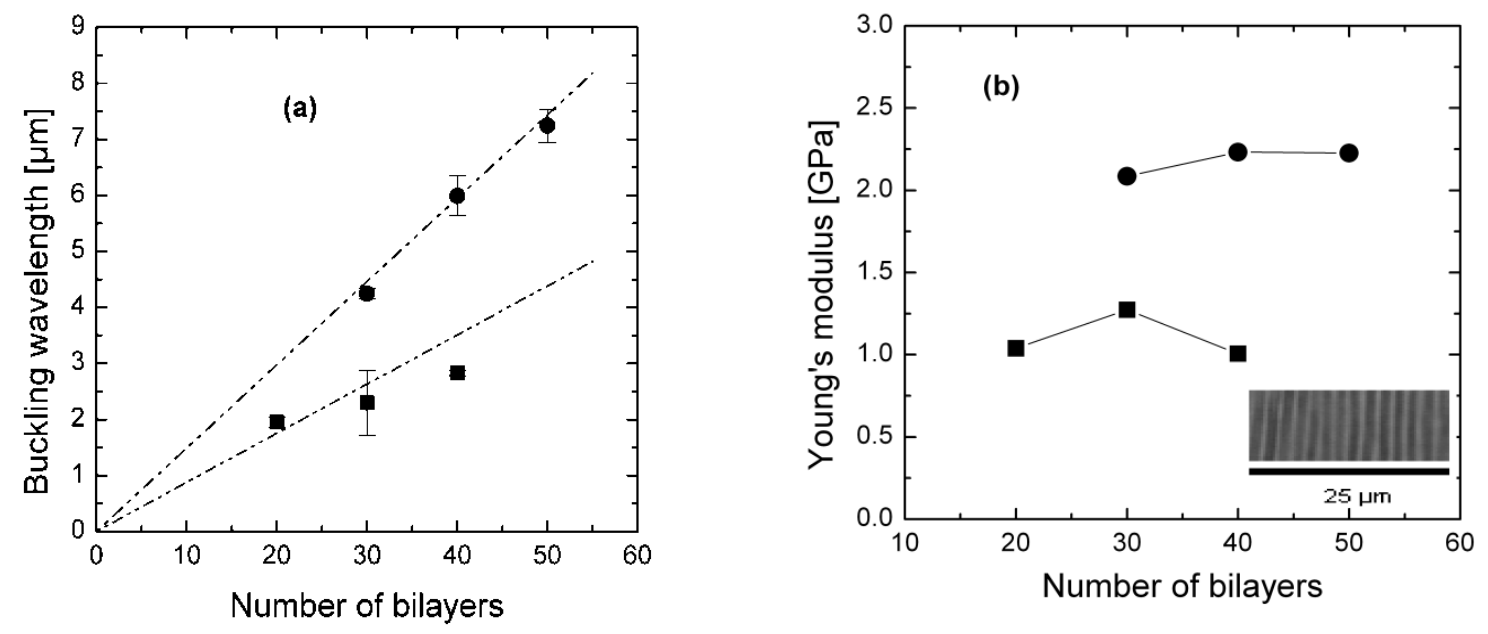

Figure 5. (a) buckling wavelength and (b) Young's modulus as a function of the number of bilayers of the PVAm-NFC (squares) and PVAm-SiO 2 -PVAm-NFC (circles) films built on PDMS substrates, the inset image presents a buckled 20 bilayers film of PVAm-NFC.

Direct UV-visible light transmittances through thin films of PVAm-NFC and PVAm-SiO ${ }_{2}$ PVAm-NFC on PDMS substrates are shown in figure 5. The PDMS reference substrate exhibits about $93 \%$ transmittance compared to air. As seen in figure 5, both systems still show transmittance close to that of the bare PDMS substrate, as it does not show any value below $84 \%$ within the visible range $400-700 \mathrm{~nm}$ for both film systems. The transmittance decreases with increasing the number of bilayers (except for 40 and 50 bilayers in Figure 5a), hence indicating increasing scattering or absorption with increasing number of film layers. Since NFC, silica, and PVAm all show very small light absorption as bulk materials, the decreasing direct transmittance with increasing number of layers can confidently be attributed to light scattering, i.e. most likely scattering at the film surface caused by its micro roughness. In figure 5 it is evident that the direct transmittance is larger for the PVAm-NFC than for the PVAm-SiO $2-\mathrm{PVAm}-\mathrm{NFC}$ film at 
the same number of bilayers (except for the outlier of 50 bilayers of PVAm-NFC). This agrees qualitatively with the surface roughness measurement values of the PVAm-NFC and the PVAm$\mathrm{SiO}_{2}$-PVAm-NFC, showing values $1.9 \mathrm{~nm}$ and $3.6 \mathrm{~nm}$ respectively.

For some optical applications, anti-reflective coatings are of interest in order to maximize the light transmitted and to minimize that reflected through a certain void [31-36]. The principle of antireflection is the destructive interference occurring between the reflected beams at the substrate-film interface and that at the film-air interface [37]. At normal incidence, a single layer antireflective coating must have a thickness that meets the quarter wavelength requirement for total antireflective properties $[32,39]$ :

$$
d_{f}=\frac{\lambda}{4 n_{f}}
$$

where $d_{f}$ is the thickness of the reflective coating, $\lambda$ is the wavelength of the incident light and $n_{f}$ is the refractive index of the film. For an ideal antireflective coating $n_{f}=\left(n_{1} n_{2}\right)^{1 / 2}$, where $n_{1}$ and $n_{2}$ are the refractive indices of air and the substrate, respectively [39]. Consequently, assuming a glass substrate with a refractive index of $\sim 1.5$, a single antireflective coating should have the refractive index $\sim 1.22$; the lowest refractive index known for a solid nonporous dielectric material is 1.35 [40]. As seen in figure S2 and S3 in the supporting information, thin films presented in this paper have refractive indices in the ranges of 1.56-1.52 and 1.46-1.45 throughout the visible wavelengths $400-700 \mathrm{~nm}$ for the PVAm-NFC and the PVAm- $\mathrm{SiO}_{2-}$ PVAm-NFC films, respectively. Thus they do not fulfill the refractive index requirement for an anti-reflective coating. However, in order to develop such a low refractive index coating, researchers have tried to introduce porosity to the coating film by e.g. the adsorption of colloidal nanoparticles on polyelectrolyte multilayers [41]. AFM images in figure 3 show that both systems have some nanopores with different sizes which may result in a low refractive index. However, the lowered refractive index due to increased porosity and the inclusion of silica particles ( 1.45) are still far away from that needed for antireflecting coatings (1.22). 

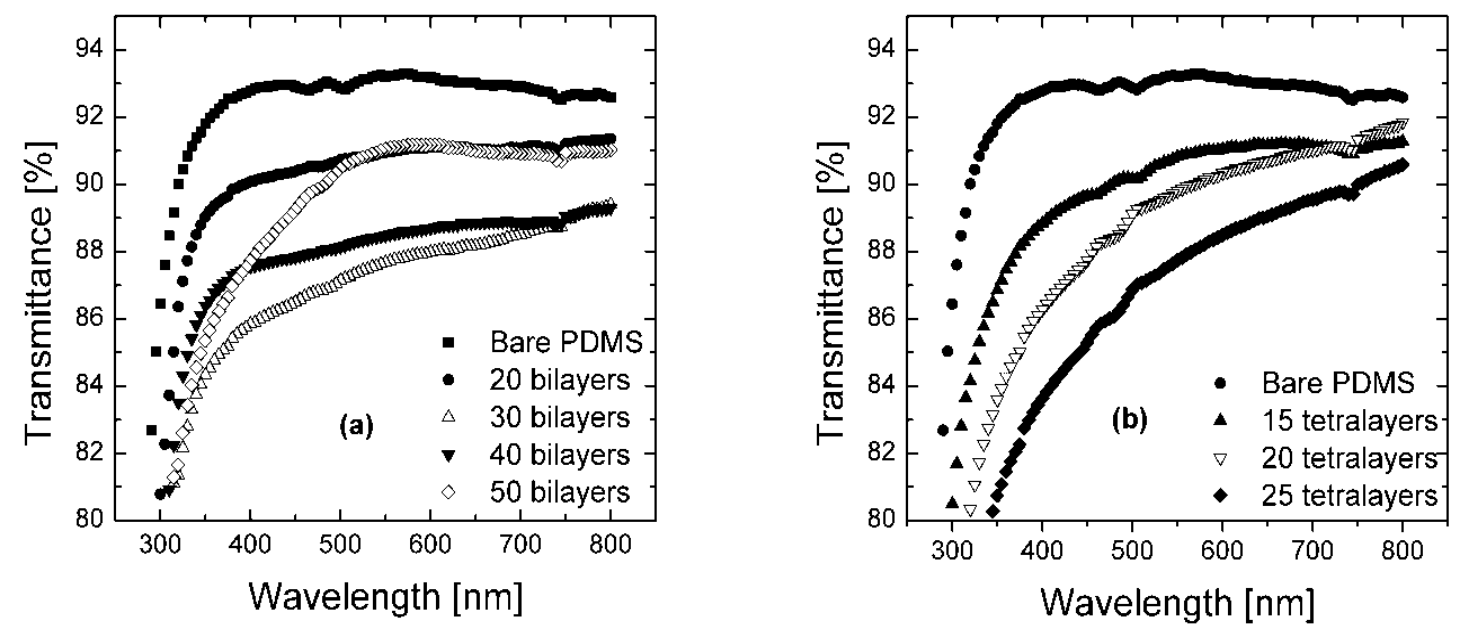

Figure 6. UV-visible transmittance of thin films of (a) PVAm-NFC and (b) PVAm-SiO ${ }_{2}-\mathrm{PVAm}-\mathrm{NFC}$ on PDMS substrates. Both systems exhibit relatively large transmittance values which decreases monotonously with increasing film thickness, except for the 50 bilayers of PVAm-NFC.

\section{Conclusion}

In conclusion, we report on the fabrication of a functional thin film based on a renewable resource material. Thin films of NFC of enhanced Young's modulus and relatively high light transmission were prepared on an elastic PDMS substrate by incorporating silica nanoparticles in the film. The multilayer build-up and the internal film structure were well characterized by combining the techniques of QCM-D, ellipsometry and AFM. Although the Young's modulus of PVAm-NFC film was found smaller than that obtained for NFC-PEI films. However, incorporating silica nanoparticles enhanced the Young's modulus from 1 to $2 \mathrm{GPa}$. Whereas PVAm played a crucial rule in affording high degree of nanoporosity, silica nanoparticles were utilized to enhance the Young's modulus of the whole film due to its own higher Young's modulus. The elasticity of PDMS, the relatively large visible light transmittance of the film as well as its enhanced Young's modulus make these films of high potential for different applications such as optical components for high performance devices, solar cells or biomedical devices. 


\section{Acknowledgement:}

We thank Erik Johansson, Institute of fibre technology, KTH, for his help with the buckling measurements and Prof. Mamoun Muhammed, functional materials division (FNM), KTH, for the UV-visible transmission measurements.

Supporting Information Available: figures S1-S3.

\section{References}

[1] S. J. Eichhorn, A. Dufresne, M. Aranguren, N. E. Marcovich, J. R. Capadona, S. J. Rowan, C. Weder, W. Thielemans, M. Roman, S. Renneckar, W. Gindl, S. Veigel, J. Keckes, H. Yano, K. Abe, M. Nogi, A. N. Nakagaito, A. Mangalam, J. Simonsen, A. S. Benight, A. Bismarck, L. A. Berglund, T. Peijs , J. Mater. Sci. 45 (2010) 1.

[2] D. Klemm, F. Kramer, S. Moritz, T. Lindström, M. Ankerfors, D. Gray, A. Dorris, Angew. Chem. Int. Ed., 50 (2011) 2-31.

[3] E.D. Cranston, D.G. Gray, Model Cellulose surfaces, M. Roman (Ed.) ACS Symposium Series 2010, 95.

[4] L. Wågberg, G. Decher, M. Norgren, T. Lindström, M. Ankerfors, K. Axnäs, Langmuir $24,(2008) 784$.

[5] H. Fukuzumi, T. Saito, T. Iwata, Y. Kumamoto, A. Isogai, Biomacromolecules 10 (2009) 162.

[6] C. Aulin, M. Gällstedt, T. Lindström, Cellulose 17 (2010) 559.

[7] M. Henriksson, L. A. Berglund, P. Isaksson, T. Lindström, T. Nishino, Biomacromolecules 9 (2008) 1579.

[8] E. Karabulut, L. Wågberg, Soft Matter 7 (2011) 3467. 
[9] C. M. Stafford, C. Harrison, K. L. Beers, K. Alamgir, E. J. Amis, M. R. Vanlandingham, H. Kim, W.Volksen, R.D. Miller, E. E. Simonyi, Nat. Mater. 3 (2004) 545.

[10] A. J. Nolte, M. F. Rubner, R. E. Cohen, Macromolecules 38 (2005) 5367.

[11] A. J. Nolte, R. E. Cohen, M. F. Rubner, Macromolecules 39 (2006) 4841.

[12] H. Huang, J. Y. Chung, A. J. Nolte, C. M. Stafford, Chem. Mater. 19 (2007) 6555.

[13] E. Cranston, M. Eita, E. Johansson, J. Netrval, M. Slajkova, H. Arwin, L. Wågberg, Biomacromolecules, 12 (2011) 961.

[14] H. G. Tompkins, E. A. Irene (Eds.), Handbook of Ellipsometry, William Andrew, New York, 2005.

[15] A. J. Nolte, N. D. Treat, R. E. Cohen, M. F. Rubner, Macromolecules 41(2008) 5793.

[16] J. Y. Chung, A. J. Nolte, C. M. Stafford, Adv. Mater. 23 (2010) 349.

[17] C. Jiang, S. Singamaneni, E. Merrick, V. V. Tsukruk, Nano Lett. 6 (2006) 2254.

[18] R. Gunawidjaja, C. Y. Jiang, S. Peleshanko, M. Ornatska, S Singamaneni, V. V. Tsukruk, Adv. Mater. 18 (2006) 829.

[19] C. Y. Jiang, X. Y. Wang, R. Gunwidjaja, Y.H. Lin, M. K. Gupta, D. L. Kaplan, R. R. Naik, V. V. Tsukruk, Adv. Funct. Mater. 17 (2007) 2229.

[20] D. Y. Khang, H. Q. Jiang, Y. Huang, J. A. Rogers, Science 311 (2006) 208.

[21] N. Bowden, S. Brittain, A. G. Evans, J. W. Hutchinson, G. M. Whitesides, Nature, 393 (1998) 146.

[22] D. Y. Khang, J. L. Xiao, C. Kocabas, S. Maclaren, T. Banks, H. Q. Jiang, Y. Y. G. Huang, J. A. Rogers, Nano Lett. 8 (2008) 124.

[23] D. S. Ballantine, R. M.White, S. I. Martin, A. J. Ricco, E. T. Zellers, G. C. Frey, H. Wohltjen, Acoustic Wave sensors: Theory, Design and Physico-chemical Applications. Academic Press Inc. 1997, Ch. 3, p 36. 
[24] G. Sauerbrey, Zeitschrift für Physik, 155 (1959) 206.

[25] M. Rodahl, F. Höök, A. Krozer, P. Brzezinski, B. Kasemo, Rev. Sci. Instrum. 66 (1995) 3924.

[26] E. Reimhult, C. Larsson, B. Kasemo, F. Höök, Anal. Chem. 76 (2004) 7211.

[27] M. Eita, Soft Matter, 7 (2011) 709.

[28] M. Eita, Soft Matter, DOI:10.1039/C1SM05806A.

[29] M. Zou, D. Yang, Tribology letters, 22 (2006) 189.

[30] H. Mahfuz, M. Hassan, V. Dhanak, G. Beamson, J. Stewart, V. Rangari, X. Wei, V. Khabashesku, S. Jeelani, Nanotechnology, 19 (2008) 445702.

[31] W. Shimizu, Y. Murakami, Appl. Mater. Interfaces, 2 (2010) 3128.

[32] B. E. Yoldas, Appl. Opt. 19 (1980) 1425.

[33] I. M. Thomson, Appl. Opt. 31 (1992) 6145.

[34] M. Ibn-Elhaj, M. Schadt, Nature 410 (2001) 796.

[35] B. G. Prevo, Y. Hwang, O. D. Velev, Chem. Mater. 17 (2005) 3642.

[36] J. -Q. Xi, J. K. Kim, E. F. Schubert, Nano Lett. 5 (2005) 1385.

[37] P. B. Clapham, M. C. Hutley, Nature 244 (1973) 281.

[38] M. Bass, Handbook of optics. Fundamentals, Techniques \&design. McGraw-Hill, New York, 1995.

[39] S. Walheim, E. Schäffer, J. Mlynek, U. Steiner, Science 283 (1999) 520.

[40] D. R. Uhlmann, T. Suratwala, K. Davidson, J. M. Boulton, G. Teowee, J. NonCryst. Solids 218 (1998) 113.

[41] H. Hattori, Adv. Mater. 13 (2001) 51. 